\title{
BMJ Open Differences in predictors of permanent work disability between immigrants and natives: a cohort study of adults with sick leave due to common mental disorders
}

\author{
Laura Werlen, ${ }^{1}$ Magnus Helgesson, ${ }^{2}$ Ellenor Mittendorfer-Rutz ${ }^{2}$
}

To cite: Werlen $L$, Helgesson M, MittendorferRutz E. Differences in predictors of permanent work disability between immigrants and natives: a cohort study of adults with sick leave due to common mental disorders. BMJ Open 2017;7:e014431. doi:10.1136/bmjopen-2016014431

- Prepublication history for this paper is available online. To view these files please visit the journal online (http://dx.doi.org/10.1136/ bmjopen-2016-014431).

Received 27 September 2016 Revised 13 February 2017 Accepted 21 February 2017

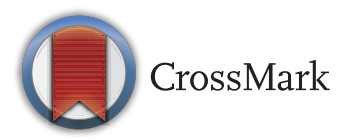

${ }^{1}$ Epidemiology, Biostatistics and Prevention Institute, University of Zurich, Zurich, Switzerland

${ }^{2}$ Division of Insurance Medicine, Department of Clinical Neuroscience, Karolinska Institutet, SE-171 77 Stockholm, Sweden

Correspondence to Dr Magnus Helgesson; magnus.helgesson@ki.se

\section{ABSTRACT}

Objectives: Immigrants with common mental disorders (CMDs) are reported to have a higher risk of disability pension (DP) compared with native residents; however, the reasons for this are not fully understood. This study aimed to investigate (1) differences in morbidity (3 measures) and socioeconomic status in native Swedes, 'Western' and 'non-Western' immigrants with CMDs and (2) interactions between morbidity and socioeconomic status and immigrant status regarding subsequent DP.

Design: The study was a prospective populationbased cohort study using national register data. Crude and multivariate HRs with $95 \%$ Cls were calculated using the Cox regression (2007-2010).

Participants: All individuals aged 18-59 with an incident sick-leave spell due to CMDs during 2006 were included in the study ( $\mathrm{N}=66$ 097). The study population was divided into 3 groups based on country of birth: (1) Sweden, (2) immigrants from 'Western' countries (EU25, Norway, Iceland, North America and Oceania) and (3) immigrants from 'non-Western' countries (east Europe, Africa, Asia and South America).

Results: Particularly, immigrants born in non-Western countries had higher levels of morbidity and lower socioeconomic status than natives ( $p>0.001)$. No significant differences in the associations between specialised psychiatric and somatic care with regard to subsequent DP were found between immigrants and native Swedes. Being prescribed more than 1 type of psychiatric medication was associated with higher HRs for DP in immigrants from Western (HR 3.34; $\mathrm{Cl} 2.3$ to 4.9) and non-Western countries (3.6; 1.9 to 6.4$)$ than in native Swedes $(2.55 ; 2.3$ to 2.8$)\left(p_{\text {interaction }}=0.003\right)$. Low education was a marginally stronger predictor for DP in non-Western immigrants than in native Swedes and Western immigrants ( $p_{\text {interaction }}=0.03$ ).

Conclusions: Morbidity measured by medication, but not by specialised healthcare, was a stronger predictor for DP in immigrants than in native Swedes, warranting scrutiny of differences in care and treatment in immigrants and native Swedes with CMDs.

\section{Strengths and limitations of this study}

- The study is a prospective cohort study using a large study population with a relatively long follow-up period of 4 years.

- The Swedish national registers used here are of good quality with almost no dropout.

- The validity of the diagnoses of sick-leave spells has often been discussed, but a previous related study reported good validity.

- Findings of this study cannot be generalised to a population with depression and anxiety symptoms that did not come to the attention of the healthcare system.

- Grouping immigrants according to regions of country of birth could mask critical differences, including country of origin and reason for emigration.

\section{INTRODUCTION}

Common mental disorders (CMDs) are a significant and growing cause of morbidity in Sweden and across the globe. By 2030, depressive disorders are predicted to be the leading diagnoses resulting in loss of disability-adjusted life years in high-income countries. ${ }^{1}$ Moreover, CMDs have a strong impact on work capacity, ${ }^{2}{ }^{3}$ putting individuals with these disorders at risk for temporal and permanent work disability (ie, sickness absence (SA) and disability pension (DP) $).^{4-6}$

To date, immigrants form a considerable proportion of the population in Sweden, and this proportion is estimated to increase in the years to come. ${ }^{78}$ Previous studies have reported immigrants to Sweden and Denmark to be at higher risk of psychiatric morbidity compared with the native population $^{9-11}$ and that there are differences between immigrants and the native 
population regarding the work-related consequences of having a CMD. For example, immigrants from non-Western countries have been found to be at higher risk for temporal and permanent work disability compared with the native population; ${ }^{6}{ }^{12}$ however, the reasons for this are not yet fully understood.

Previously reported risk factors for an increased risk of DP in individuals with a CMD are the severity of the underlying mental disorder, a comorbid somatic disorder and low socioeconomic status. ${ }^{56}$ However, it is not known whether these patterns differ between immigrants and the native population. Adding to the complexity of this question, migration is not a homogenous phenomenon, so the pathways to permanent work disability might vary for different immigrant groups with respect to country of birth. Especially, immigrants from non-Western countries have been shown to have a higher prevalence of CMDs and work disability. ${ }^{6}{ }^{12}$ It is therefore important to consider different immigrant groups in studies on this topic. In this study, immigrants were divided into Western immigrants, a group mostly consisting of voluntary immigrants, for example, labour migrants and students, and non-Western immigrants, a group largely comprised refugees and relatives seeking reunification with refugees. ${ }^{13}$ The pathways to DP are not only strongly affected by a number of other sociodemographic factors, for example, age, sex and type of living area in Sweden, but also by previous spells of unemployment and/or sick leave. ${ }^{14}$ Therefore, further consideration of these factors in related studies is crucial. Moreover, due to the reported differences in access to healthcare as well as in diagnostics and treatment in immigrants compared with the native population, different measures of morbidity should be used in studies that include immigrants. ${ }^{9}$

\section{Aim}

This study aimed to describe: (1) differences in morbidity and/or socioeconomic status with regard to immigrant status in adults with temporary work disability due to CMDs and (2) interactions between morbidity and socioeconomic status and immigrant status regarding subsequent permanent work disability among individuals with temporary work disability due to a CMD.

\section{METHODS}

\section{Study population}

This study is a prospective, population-based cohort study including all non-pensioned individuals aged 18-59 years and living in Sweden on 31 December 2005 who had a new, incident sick-leave spell due to a CMD during 2006 $(\mathrm{N}=66$ 097). Individuals were followed regarding DP from 1 January 2007 until 31 December 2010.

Using the unique deidentified personal identification number for all Swedish residents, data on individuals were linked at the individual level. These data were obtained from registers from the following three agencies: (1) Statistics Sweden: the Longitudinal Integration Database for Health Insurance and Labour Market Studies (LISA) containing information on age, sex, country of birth, educational level, type of living area, family composition and length of unemployment. (2) The Social Insurance Agency: the Micro Data for Analyses of Social Insurance (MiDAS) register including information on the date, grade and diagnoses of SA and DP. (3) The National Board of Health and Welfare: the National Patient Register (NPR) comprising data on the date and cause of inpatient and specialised outpatient healthcare from 1973 and from 2001, respectively; the Cause of Death Register (CDR) with information on the date of death from 1960 and onwards; and the Prescribed Drug Register (PDR) with information on the prescription of dispensed psychiatric medication (date of dispensing and type), from July 2005 and onward.

\section{The Swedish social insurance system}

Swedish sickness insurance covers all people above the age of 16 who are living in Sweden and have at least a minimum annual income from work. ${ }^{15}$ Compensation can be provided for individuals with reduced working capacity of at least $25 \%$ due to a disease or injury by either the employer or the Social Insurance Agency (SIA). Employees receive sick pay from days 2 to 14 of a sick-leave spell from the employer (the first day being a qualifying day). From day 15, employees get sickness benefit from SIA. A certificate from a physician is required from the eighth day of a sick-leave spell. Unemployed individuals and individuals on parental leave can be granted sickness benefit from SIA from the second day of a sick-leave spell, whereas self-employed individuals receive sick pay from SIA according to which insurance coverage they had chosen. In Sweden, DP can be granted to all individuals living in Sweden whose work capacity has been reduced due to a disease or injury. Individuals between 19 and 29 years can be granted time-restricted DP due to impaired work capacity and if they have not completed their compulsory education at 19 years of age.

\section{Variables}

The study population was divided into three groups based on country of birth: (1) Sweden (natives), (2) immigrants from 'Western' countries consisting of EU25 plus Norway and Iceland, North America and Oceania and (3) immigrants from all remaining countries, labelled as 'non-Western' countries (Europe outside EU25 and Nordic countries, Africa, Asia and South America).

The outcome measure was defined as having been granted all-cause DP in the period from 2007 through 2010 .

Morbidity was conceptualised using three different measures, namely: (1) specialised inpatient and outpatient healthcare due to mental disorders (2001-2006), (2) 
specialised inpatient and outpatient healthcare due to somatic disorders (2001-2006) and (3) psychiatric medication in 2006 grouped as (I) no medication, (II) antidepressants only, (III) anxiolytics/hypnotics/sedatives only and (IV) more than one of these types of psychiatric medication in any combination. Moreover, socioeconomic status was measured as educational level in 2005 divided into (I) low education level (through elementary school; $\leq 9$ years), (II) medium education level (high school, 10-12 years) and (III) high education level (university; $>12$ years). Missing data regarding education were treated as low educational level.
Covariates included age, sex, type of living area, family composition as well as previous unemployment and previous $\mathrm{SA}$, which were measured for the year preceding baseline and coded as indicated in table 1 .

\section{Diagnostics and codes}

All diagnoses were coded according to the International Classification of Diseases V.10 (ICD 10) codes. Here, CMDs were categorised into three diagnostic groups: (1) depressive disorders (F32: depressive episode and F33: recurrent depressive disorder), (2) anxiety disorders (F40: phobic anxiety disorders, F41: other anxiety

Table 1 Descriptive statistics of the 66097 women and men, aged 18-59 years on 31 December 2005 with at least one new sick-leave spell due to a common mental disorder (CMD) in 2006 in Sweden and the share with disability pension between 2007 and 2010

\begin{tabular}{|c|c|c|c|c|}
\hline & \multicolumn{2}{|l|}{ All } & \multicolumn{2}{|l|}{ DP } \\
\hline & $\mathbf{N}$ & Per cent & $\mathbf{N}$ & Per cent \\
\hline Study population, all & 66097 & 100 & 4610 & 7.0 \\
\hline \multicolumn{5}{|l|}{ Country of birth* } \\
\hline Sweden & 57011 & 86.3 & 3696 & 6.5 \\
\hline Western countries & 3520 & 5.3 & 302 & 8.6 \\
\hline Non-Western countries & 5566 & 8.4 & 612 & 11.0 \\
\hline \multicolumn{5}{|l|}{ Sex } \\
\hline Male & 20373 & 30.8 & 1540 & 7.6 \\
\hline Female & 45724 & 69.2 & 3070 & 6.7 \\
\hline \multicolumn{5}{|l|}{ Age in 2005} \\
\hline $18-24$ & 4420 & 6.7 & 295 & 6.7 \\
\hline $25-34$ & 17029 & 25.8 & 731 & 4.3 \\
\hline $35-44$ & 21228 & 32.1 & 1151 & 5.4 \\
\hline $45-54$ & 16516 & 25.0 & 1363 & 8.3 \\
\hline $55-59$ & 6904 & 10.4 & 1070 & 15.5 \\
\hline \multicolumn{5}{|l|}{ Type of living area† } \\
\hline Big cities & 25450 & 38.5 & 1610 & 6.3 \\
\hline Medium-sized cities & 23186 & 35.1 & 1585 & 6.8 \\
\hline Small cities/villages & 17461 & 26.4 & 1415 & 8.1 \\
\hline \multicolumn{5}{|l|}{ Family composition } \\
\hline Married/cohabiting with children at home & 26976 & 40.8 & 1434 & 5.3 \\
\hline Married/cohabiting without children at home & 6792 & 10.3 & 690 & 10.2 \\
\hline Single $\ddagger$ with children at home & 9579 & 14.5 & 628 & 6.6 \\
\hline Single $\ddagger$ without children at home & 22750 & 34.4 & 1858 & 8.2 \\
\hline \multicolumn{5}{|l|}{ Unemployment in 2005} \\
\hline No days & 54637 & 82.7 & 3276 & 6.0 \\
\hline $1-90$ days & 5435 & 8.2 & 500 & 9.2 \\
\hline$>90$ days & 6025 & 9.1 & 834 & 13.8 \\
\hline \multicolumn{5}{|l|}{ Sick leave in 2005} \\
\hline No days & 48749 & 73.8 & 2769 & 5.7 \\
\hline $1-90$ days & 13944 & 21.1 & 1309 & 9.4 \\
\hline$>90$ days & 3404 & 5.2 & 532 & 15.6 \\
\hline \multicolumn{5}{|l|}{ Sick leave diagnosis in 2006} \\
\hline Depressive & 24419 & 36.9 & 2296 & 9.4 \\
\hline Anxiety & 7827 & 11.8 & 806 & 10.3 \\
\hline Stress-related & 33851 & 51.2 & 1508 & 4.5 \\
\hline
\end{tabular}

${ }^{*}$ Country of birth categorised according to: (1) Sweden, (2) Western countries include the Nordic countries (Denmark, Finland, Iceland and Norway), EU25 (all countries included in the European Union in 2006 without Sweden), North America and Oceania, (3) non-Western countries comprise all remaining countries.

†Type of living area: big cities, Stockholm, Gothenburg and Malmo; medium-sized cities, cities with >90 000 inhabitants within 30 km distance from the centre of the city; small cities/villages, all remaining cities/villages.

‡Single means living without partner and also includes divorced, separated or widowed. 
Table 2 Descriptive statistics of 66097 women and men, aged 18-59 years on 31 December 2005 with at least one new sick-leave spell due to a common mental disorder in 2006 in Sweden with regard to morbidity and socioeconomic status, stratified by migration status

\begin{tabular}{|c|c|c|c|c|}
\hline & $\begin{array}{l}\text { Sweden } \\
\text { N (\%) }\end{array}$ & $\begin{array}{l}\text { Western countries* } \\
\text { N (\%) }\end{array}$ & $\begin{array}{l}\text { Non-Western countriest } \\
\mathrm{N}(\%)\end{array}$ & Significance testł \\
\hline \multicolumn{5}{|c|}{ Specialised healthcare 2001-2006 } \\
\hline \multicolumn{5}{|c|}{ Mental diagnosis } \\
\hline None & $44612(78.3)$ & $2748(78.1)$ & 4073 (73.2) & $\chi 2=75.8$ \\
\hline Any & $12399(21.7)$ & $772(21.9)$ & $1493(26.8)$ & $\mathrm{p}<0.001$ \\
\hline \multicolumn{5}{|l|}{ Somatic diagnosis } \\
\hline None & 9509 (16.7) & 569 (16.2) & $690(12.4)$ & $\chi 2=68.2$ \\
\hline Any & 47502 (83.3) & $2951(83.8)$ & $4876(87.6)$ & $\mathrm{p}<0.001$ \\
\hline \multicolumn{5}{|c|}{ Psychiatric medication§ 2006} \\
\hline None & 21049 (36.9) & $1185(33.7)$ & $1800(32.3)$ & $\chi 2=145.6$ \\
\hline Antidepr only & 10918 (19.2) & $618(17.6)$ & 903 (16.2) & $p<0.001$ \\
\hline Hyp/Sed/Anx only & $6413(11.2)$ & $416(11.8)$ & $684(12.3)$ & \\
\hline Mixed medications & 18631 (32.7) & $1301(37.0)$ & 2179 (39.1) & \\
\hline \multicolumn{5}{|l|}{ Socioeconomic status } \\
\hline \multicolumn{5}{|l|}{ Education, 2005} \\
\hline Low & $6982(12.2)$ & $546(15.5)$ & 1071 (19.2) & $\chi 2=263.8$ \\
\hline Medium & 29960 (52.6) & $1645(46.7)$ & 2697 (48.5) & $p<0.001$ \\
\hline High & 20069 (35.2) & 1329 (37.8) & 1798 (32.3) & \\
\hline \multicolumn{5}{|c|}{ 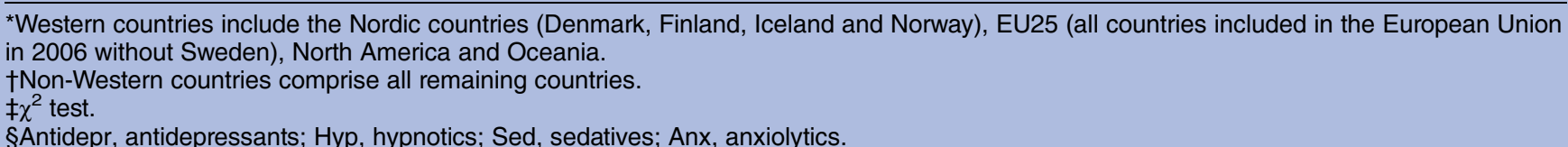 } \\
\hline
\end{tabular}

disorders and F42: obsessive-compulsive disorder) and (3) stress-related disorders (F43: reaction to severe stress and adjustment disorders). Mental (F00-99) and somatic (all other ICD codes) diagnoses were used to measure inpatient and specialised outpatient healthcare. Information on psychiatric medication was categorised according to the Anatomical Therapeutic Chemical (ATC) classification system: antidepressants (ATC: N06A); anxiolytics, sedatives and hypnotics (ATC: N05B or N05C).

\section{Statistical methods}

Pearson's $\chi^{2}$ tests were performed in order to examine differences between immigrant groups and native Swedes regarding measures of morbidity and socioeconomic status. Crude and multivariate HRs and CIs regarding the association between morbidity and socioeconomic factors and subsequent granting of DP were calculated by the Cox proportional hazard regression after assuring that the proportional hazard assumption was met. These analyses were stratified by immigrant status, that is, native Swedes and immigrants born in Western and non-Western countries. Censoring in the regression analyses was due to emigration, death or end of follow-up, whichever came first. Multivariate analyses were adjusted for all variables, that is, measures of morbidity, socioeconomic status, sociodemographics, previous unemployment, SA and diagnoses of SA in 2006 (all variables mentioned in tables 1 and 2). Partial likelihood ratio tests were performed in order to test potential interactions between the different measures of morbidity and socioeconomic status in addition to immigrant status and subsequent granting of DP. Analyses were conducted using IBM SPSS Statistics V.22.0.

\section{RESULTS}

During the 4 years of follow-up, $7 \%$ of individuals with SA due to CMDs were granted DP (table 1).

There was an obvious gradient showing that immigrants from non-Western countries $(11.0 \%)$ had the highest rate of DP followed by immigrants from Western countries $(8.6 \%)$ and native Swedes $(6.5 \%)$. More than two-thirds of individuals with at least one new sick-leave spell due to a CMD in 2006 were women (table 1). Still, a slightly higher proportion of men $(7.6 \%)$ was granted DP during follow-up when compared with women (6.7\%). Age was related to subsequent DP in a J-shaped fashion (table 1). Moreover, DP was more common among persons living in small cities/villages, married/cohabiting without children living at home and those with long earlier periods of unemployment and/or SA. The proportion of individuals who were subsequently granted DP was highest among those with anxiety disorders followed by depressive disorders and stress-related disorders (table 1).

There were significant differences in the proportion of all markers of morbidity and socioeconomic status across immigrant groups and native Swedes. Immigrants from non-Western countries had higher morbidity expressed as a higher prevalence of mental $(\mathrm{p}<0.001)$ and somatic $(p<0.001)$ specialised healthcare compared 
with native Swedes and immigrants from Western countries (table 2).

Moreover, a higher proportion of immigrants from outside the Western world had a higher prescription rate of more than one type of psychiatric medication compared with native Swedes and lower rates of being prescribed only antidepressants $(p<0.001)$. Immigrants from non-Western countries also had a significantly higher prevalence of low educational level compared with native Swedes $(p<0.001)$. There were fewer differences between native Swedes and immigrants from the Western world (table 2).

The univariate and multivariate models show that the HRs of being granted DP during follow-up after having received specialised healthcare due to mental diagnoses were higher for all groups, while the HRs of DP related to previous specialised healthcare due to somatic diagnoses were increased only among native Swedes (table 3).

The risk for DP in the event of psychiatric healthcare was around twofold higher than the risk without such care in all groups. No significant interaction was observed between diagnosis-specific healthcare measures and immigrant group and subsequent granting of DP.

The HRs of DP were higher among individuals in all groups when prescribed only antidepressants or more than one type of psychiatric medication compared with no prescribed psychiatric medication (table 3). Native Swedes and immigrants from the Western world also had higher HRs for DP when they had been prescribed hypnotics/sedatives/anxiolytics only. There was a significant interaction between psychiatric medication and immigrant status with regard to subsequent granting of DP $(p=0.003)$. In the multivariate analyses, the HRs of DP related to having more than one type of psychiatric medication were twofold and threefold higher in native Swedes and immigrants, respectively (table 3 ).

Individuals in all groups with low educational level had higher HRs for DP in the univariate models (table $3)$. Interactions of education and immigrant status were significant $(\mathrm{p}=0.026)$. In the multivariate model, low educational level was associated with a $30 \%$ and $90 \%$ increased risk of DP for natives and immigrants from non-Western countries, respectively.

\section{DISCUSSION}

\section{Main findings}

In a population of individuals with SA due to CMDs, higher proportions of immigrants from Western (8.6\%) and non-Western $(11.0 \%)$ countries were subsequently granted DP during follow-up compared with native Swedes $(6.5 \%)$. Immigrants from non-Western countries had higher levels of morbidity and lower socioeconomic status than natives and immigrants from Western countries. Morbidity measured by being prescribed more than one type of psychiatric medication, but not as specialised psychiatric healthcare, was a stronger predictor for DP in immigrants than in natives.
Compared with native Swedes, a higher proportion of immigrants, particularly those born in non-Western countries, was granted DP during follow-up in individuals with sick leave due to CMDs. This finding is in line with previous studies on psychiatric outpatients with depressive disorders and people with SA due to stressrelated disorders. ${ }^{12} 16$ This study also found significant differences in measures of morbidity with regard to immigrant status in individuals with SA due to CMDs. Immigrants from non-Western countries in particular had higher rates of morbidity than native Swedes. This is also in line with previous studies showing more psychiatric and somatic morbidity in immigrants from outside Europe. ${ }^{17}$ In this study, morbidity was conceptualised in three different ways, that is, by specialised psychiatric and somatic healthcare as well as having been prescribed specific psychiatric medication. These measures can be regarded as reflecting the varying medical severity of the underlying mental disorders. Higher rates of specialised healthcare and being prescribed more than one type of psychiatric medication observed in immigrants from countries other than the Western world may thus be seen as an indicator of more severe morbidity, possibly due to a high proportion of refugees in this group. 5

Moreover, this study found significant interactions between morbidity and immigrant status with regard to subsequent granting of DP in individuals with SA due to CMDs. Being prescribed more than one type of psychiatric medication was associated with higher risk estimates in immigrants than in native Swedes. Such interactions were not found when morbidity was measured as specialised psychiatric and somatic healthcare. These discrepancies may not only be a reflection of differences in the severity of the underlying morbidity, but may also reflect differences in access and acceptance of specialised healthcare, culturally sensitive diagnostics, reporting of mental symptoms and treatment in terms of psychiatric medication. ${ }^{17-21}$ Preconceptions on the part of healthcare professionals could also contribute to differential treatment. ${ }^{1922}$ Future studies are warranted to elucidate potential differences in immigrant groups and native Swedes with SA due to CMDs regarding the type of treatment provided and the role of healthcare in preventing transition from temporal to permanent work disability.

In line with previous studies, this study also shows that immigrants, particularly from non-Western countries, had lower levels of education compared with native Swedes. ${ }^{17}$ We could now also report that low educational level was a stronger predictor for subsequent DP in immigrants from non-Western countries than for natives. Potential explanations include differences in access to labour market and social insurance measures among immigrants with a culture that differs from the new country, but may also be explained by discrimination. ${ }^{23-25}$ The share of individuals with a high level of education is generally around the same among non-Western immigrants as among native Swedes. ${ }^{26}$ The most striking discrepancy in these two 
Table 3 Univariate and multivariate* HRs, with 95\% Cls, for being granted disability pension (2007-2010) of the 66097 women and men, aged 18-59 years on 31 December 2005 with at least one sickness absence spell due to a common mental disorder in 2006, stratified by migration status

\begin{tabular}{|c|c|c|c|c|c|c|c|c|}
\hline \multicolumn{3}{|c|}{ Sweden } & \multicolumn{3}{|c|}{ Western countries $†$} & \multicolumn{3}{|c|}{ Non-Western countries $¥$} \\
\hline$\%$ DP & $\begin{array}{l}\text { Univariate } \\
\text { (Cl 95\%) }\end{array}$ & $\begin{array}{l}\text { Multivariate } \\
\text { (Cl 95\%) }\end{array}$ & $\%$ DP & $\begin{array}{l}\text { Univariate } \\
\text { (Cl 95\%) }\end{array}$ & $\begin{array}{l}\text { Multivariate } \\
\text { (Cl 95\%) }\end{array}$ & $\%$ DP & $\begin{array}{l}\text { Univariate } \\
\text { (Cl 95\%) }\end{array}$ & $\begin{array}{l}\text { Multivariate } \\
\text { (Cl 95\%) }\end{array}$ \\
\hline
\end{tabular}

Morbidity

Specialised healthcare 2001-2006

Mental diagnosis§

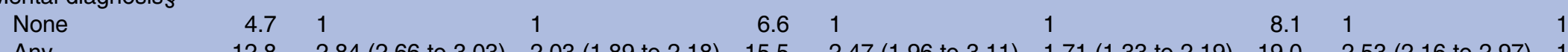

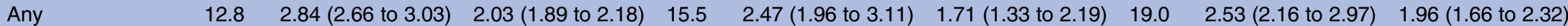

Somatic diagnosis

$\begin{array}{llllllllll}\text { None } & 4.3 & 1 & 1 & 7.0 & 1 & 1 & 1 & 1\end{array}$

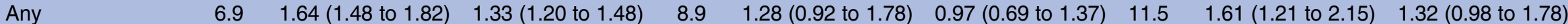

Psychiatric medication** 2006

$\begin{array}{lllllllll}\text { None } & 3.1 & 1 & 1 & 2.9 & 1 & 1 & 3.9 & 1\end{array}$

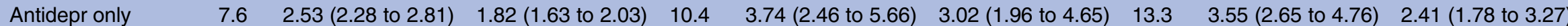

Hyp/Sed/Anx only $\quad 4.5 \quad 1.49(1.29$ to 1.71$) \quad 1.22(1.06$ to 1.40$) \quad 5.5 \quad 1.96(1.15$ to 3.32$) \quad 1.72(1.01$ to 2.93$) \quad 5.3 \quad 1.35(0.90$ to 2.01$) \quad 1.21(0.81$ to 1.81$)$

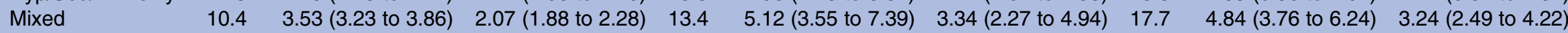

medicationst†

Socioeconomic status

Education $\$ 2005$

\begin{tabular}{|c|c|c|c|c|c|c|c|c|c|}
\hline Low & 9.4 & 1.75 (1.59 to 1.92$)$ & 1.30 (1.18 to 1.43$)$ & 11.5 & 1.75 (1.27 to 2.41$)$ & 1.31 (0.94 to 1.82$)$ & 14.8 & 1.79 (1.43 to 2.23$)$ & 1.91 (1.52 to 2.40$)$ \\
\hline Medium & 6.5 & 1.18 (1.10 to 1.27$)$ & 1.06 (0.98 to 1.14$)$ & 9.0 & 1.32 (1.02 to 1.72$)$ & 1.16 (0.89 to 1.51$)$ & 11.2 & 1.33 (1.09 to 1.61$)$ & 1.56 (1.28 to 1.90$)$ \\
\hline High & 5.5 & 1 & 1 & 6.8 & 1 & 1 & 8.5 & 1 & 1 \\
\hline
\end{tabular}

*All variables were mutually adjusted for all other variables in the table and additionally for sex, age, type of living area, family composition, unemployment in 2005 , sick leave in 2005 and sick leave diagnose in 2006.

†Western countries include the Nordic countries (Denmark, Finland, Iceland and Norway), EU25 (all countries included in the European Union in 2006 without Sweden), North America and

Oceania.

‡Non-Western countries comprise all remaining countries.

$\S$ Inpatient and/or outpatient specialised care for a mental diagnosis; $p$ value for interaction $=0.402$.

IInpatient and/or outpatient specialised care for a somatic diagnosis; $p$ value for interaction $=0.198$

${ }_{* \star}^{*}$ Antidepr, antidepressants; Hyp, hypnotics; Sed, sedatives; Anx, anxiolytics; $p$ value for interaction $=0.003$.

††Any combination of antidepressants, hypnotics, sedatives and anxiolytics.

$\ddagger \ddagger p$ Value for interaction $=0.026$ 
populations is that the share of individuals with a low level of education is much higher among non-Western immigrants, and that many of them, especially women, have difficulties with reading and writing. ${ }^{26}$ This might be one explanation for why low educational level among immigrants more often leads to permanent work disability. There is also literature suggesting that the educational level of immigrants in Sweden is under-reported to some extent. $^{26}$

\section{Strengths and limitations}

Strengths of this study include the population-based and prospective cohort design using a large study population. In addition, the follow-up period of 4 years was relatively long. The Swedish national registers used here are of good quality with almost no dropout, and missing data are also rare. ${ }^{27} 28$ The validity of the diagnoses of sick-leave spells has often been discussed, but a previous related study reported good validity. ${ }^{29}$ This study was also subject to some limitations. Findings of this study cannot be generalised to a population with depression and anxiety symptoms that did not come to the attention of the healthcare system. Previous studies have found barriers to seeking and accessing mental healthcare among immigrants, including cultural views on mental disorders and discrimination in the host country. ${ }^{19} 2425$ This may lead to only the most severe cases coming to the attention of the healthcare system; in this case, immigrants' risk of DP in the case of specialised healthcare would be underestimated compared with that of native Swedes. Finally, immigrant groups to Sweden are very diverse. Owing to restrictions in our database, we unfortunately do not have data on specific country of birth or reason for immigration. Grouping immigrants by region of country of birth, that is, into Western and non-Western immigrants, could mask critical differences such as reason for migration. ${ }^{9}$

\section{CONCLUSION}

Prescribed psychiatric medication, but not specialised healthcare, was a stronger predictor for permanent work disability in immigrants than in native Swedes. This warrants scrutiny of differences in care and treatment of immigrants and native Swedes with CMDs in the disabling process finally resulting in permanent work disability.

Contributors LW, MH and EM-R conceived and designed the study, interpreted the data and wrote and edited the manuscript. EM-R was the guarantor of this work. LW, MH and EM-R reviewed and approved the final version submitted for publication.

Funding This study was funded by the Swedish Research Council for Health, Working Life and Welfare, grant number 2015-00742.

Competing interests None declared.

Ethics approval Regional ethical review board in Stockholm, Sweden.

Provenance and peer review Not commissioned; externally peer reviewed.

Data sharing statement We use data from three Swedish governmental authorities, Statistics Sweden, The Swedish Insurance Agency and the
Swedish National Board of Health and Welfare. Data are available to research projects that have ethical approval from a regional ethics review board.

Open Access This is an Open Access article distributed in accordance with the Creative Commons Attribution Non Commercial (CC BY-NC 4.0) license, which permits others to distribute, remix, adapt, build upon this work noncommercially, and license their derivative works on different terms, provided the original work is properly cited and the use is non-commercial. See: http:// creativecommons.org/licenses/by-nc/4.0/

\section{REFERENCES}

1. Whiteford HA, Degenhardt L, Rehm J, et al. Global burden of disease attributable to mental and substance use disorders: findings from the Global Burden of Disease Study 2010. Lancet 2013;382:1575-86.

2. Lerner D, Henke RM. What does research tell us about depression, job performance, and work productivity? J Occup Environ Med 2008;50:401-10.

3. Simon GE, Barber C, Birnbaum HG, et al. Depression and work productivity: the comparative costs of treatment versus nontreatment. J Occup Environ Med 2001;43:2-9.

4. Bültmann U, Christensen KB, Burr H, et al. Severe depressive symptoms as predictor of disability pension: a 10-year follow-up study in Denmark. Eur J Public Health 2008;18:232-4.

5. Ishtiak-Ahmed K, Perski A, Mittendorfer-Rutz E. Risk markers of all-cause and diagnosis-specific disability pension-a prospective cohort study of individuals sickness absent due to stress-related mental disorders. BMC Public Health 2014;14:805.

6. Mittendorfer-Rutz E, Härkänen T, Tiihonen J, et al. Association of socio-demographic factors, sick-leave and health care patterns with the risk of being granted a disability pension among psychiatric outpatients with depression. PLoS ONE 2014;9:e99869.

7. Arslan C. A New Profile of Migrants in the Aftermath of the Recent Economic Crisis. OECD Social, Employment and Migration Working Papers, OECD Publishing, 2014;No. 160.

8. Migrationsverket. Verksamhets- och utgiftsprognos. (The Swedish Migration Agency: Operating and expenditure forecast) [In Swedish] Februari. 2015.

9. Gilliver SC, Sundquist J, Li X, et al. Recent research on the mental health of immigrants to Sweden: a literature review. Eur J Public Health 2014;24:72-9.

10. Tinghög $\mathrm{P}, \mathrm{Al}-\mathrm{Saffar} \mathrm{S}$, Carstensen J, et al. The association of immigrant- and non-immigrant-specific factors with mental ill health among immigrants in Sweden. Int J Soc Psychiatry 2010;56:74-93.

11. Barghadouch A, Carlsson J, Norredam M. Psychiatric disorders and predictors hereof among refugee children in early adulthood: a register-based cohort study. J Nerv Ment Dis 2016. [Epub ahead of print 1 Aug 2016]

12. Ishtiak-Ahmed K, Perski A, Mittendorfer-Rutz E. Predictors of suicidal behaviour in 36,304 individuals sickness absent due to stress-related mental disorders-a Swedish register linkage cohort study. BMC Public Health 2013;13:492.

13. Migration and Asylum 2005-2015; Residence permits granted 19802015; Residence permits granted 1980-2014-refugees; Residence permits granted 1986-2014-family reunification; Residence permits granted 1986-2014-labour market; Residence permits granted 1986-2014-students; Overview and time series. Swedish Migration agency (Migrationsverket). https://www.migrationsverket.se/English/ About-the-Migration-Agency/Facts-and-statistics-/Statistics/ Overview-and-time-series.html

14. Helgesson M, Johansson B, Nordqvist $\mathrm{T}$, et al. Sickness absence at a young age and later sickness absence, disability pension, death, unemployment and income in native Swedes and immigrants. Eur $J$ Public Health 2015;25:688-92.

15. Social Insurance Agency (SIA). Social insurance in figures. Stockholm 2015. ISBN 978-91-7500-386-6.

16. Mittendorfer-Rutz E, Kjeldgard L, Runeson B, et al. Sickness absence due to specific mental diagnoses and all-cause and cause-specific mortality: a cohort study of 4.9 million inhabitants of Sweden. PLOS ONE 2012;7:e45788.

17. Hjern A. Migration and public health Health in Sweden: The National Public Health Report 2012. Chapter 13. Scand J Public Health 2012;40:255-67.

18. Kirmayer LJ, Narasiah L, Munoz M, et al. Common mental health problems in immigrants and refugees: general approach in primary care. CMAJ 2011;183:E959-67.

19. Baarnhielm S, Javo C, Mosko MO. Opening up mental health service delivery to cultural diversity: current situation, development 
and examples from three northern European countries. Adv Psychosom Med 2013;33:40-55.

20. Klinthäll M. Ethnic background, labour market attachment and severe morbidity: hospitalisation among immigrants in Sweden 1990-2001. Int Migr Integr 2008;9:45-61.

21. Meershoek A, Krumeich A. Multiculturalism and the construction of ethnic identities in labour and health practices: avoiding the culturalistic fallacy in applied research. Health Care Anal 2009;17:173-97.

22. Hjern A. High use of sedatives and hypnotics in ethnic minorities in Sweden. Ethn Health 2001;6:5-11.

23. Claussen B, Smeby L, Bruusgaard D. Disability pension rates among immigrants in Norway. J Immigr Minor Health 2012;14:259-63.

24. Pascoe EA, Smart Richman L. Perceived discrimination and health: a meta-analytic review. Psychol Bull 2009;135:531-54.
25. Hatch SL, Gazard B, Williams DR, et al. Discrimination and common mental disorder among migrant and ethnic groups: findings from a South East London Community sample. Soc Psychiatry Psychiatr Epidemiol 2016;51:689-701.

26. OECD. Finding the way: a discussion of the Swedish migrant integration system. OECD International Migration Division, Directorate for Employment, Labour and Social Affairs. July 2014.

27. Ludvigsson JF, Andersson E, Ekbom A, et al. External review and validation of the Swedish national inpatient register. BMC Public Health 2011;11:450.

28. Statistiska Centralbyrån (SCB). Årsredovisning 2014 (Statistics Sweden - Annual Report 2014) [In Swedish] Statistics Sweden 2015.

29. Ljungdahl LO, Bjurulf $P$. The accordance of diagnoses in a computerized sick-leave register with doctor's certificates and medical records. Scand J Public Health 1991;19:148-53. 